\title{
Simulation of flow field in steam ejector and analysis of shock wave characteristics
}

\author{
Yi Cui \\ Shanghai Aircraft Design and Research Institute, Commercial Aircraft Corporation of China Ltd, 5188 Jinke Road, Shanghai, \\ China
}

\begin{abstract}
Fluent software is used to simulate the two-dimensional flow field in the steam ejector. The generation, development and end of shock in the flow field are analyzed. The influence of the shock wave characteristics on the ejector performance is discussed. The results show that the working back pressure in the steam ejector and the throat diameter of the nozzle all affect the position and intensity of the shock wave. When the shock wave ends prematurely or belatedly, it all affect the performance of the injector.
\end{abstract}

\section{Introduction}

Although the structure of the steam ejector is simple, the supersonic mixing process of the gas inside the equipment is very complicated, and there are extremely complicated flow phenomena such as turbulence, entrainment, mixing, and shock waves. Wang et al. analyzed the influence of suction pressure on the pumping performance of the steam jet pump and found that the performance of the jet pump is closely related to the pressure of the pumped gas. The simulation results show that the ejector coefficient increases with the pressure of the pumped gas [1]. Xinzhuang Wu etc. analyzed the influence of working back pressure on the ejector performance and found that critical back pressure exists at the ejector outlet. Simulation results show that when the working back pressure changes, the internal shock position of the ejector also changes. It has a certain influence on the performance of the injector, but it does not do a deep research on the impact of the shock wave position on the ejection coefficient [2]. Shah etc. analyzed the effect of nozzle outlet location and mixing section structure on ejector performance. The results show that different angles of contraction can result in a larger difference in ejector ejection coefficient [3].Wu etc. analyzed the length of the mixing section and the influence of the shrinkage angle. The simulation results show that the length and the contraction angle of the mixing section have optimal values and the entrainment ratio is the largest [4]. However, the impact of the position of the shock wave in the injector on the performance of the injector has not been analyzed. The steam ejector working fluid generates a series of shock waves by generating supersonic fluid through the Laval nozzle, and position of the shock wave greatly influence the performance of the ejector. Research on the shock wave characteristics of steam ejectors has important theoretical significance and engineering value for improving the entrainment ratio of steam ejectors.

In this paper, a two-dimensional axisymmetric model of the steam ejector was established, and we simulated the complex flow inside the injector. On this basis, the effect of the back pressure of the injector and the throat diameter of the nozzle on the ejection performance was analyzed and the effects of complex flow conditions such as shock waves inside the steam ejector on the performance of the jet pump were discussed in detail.

\section{The working principle of the steam ejector}

\subsection{The working principle of the steam ejector}

The steam ejector consists mainly of the nozzle, the suction chamber, the mixing chamber, the ejector throat and diffuser, as shown in Figure 1. Its working principle is that the high pressure working steam adiabatically expands through the nozzle, converts the pressure energy into kinetic energy, and ejects it at a high speed. The pressure is reduced from $\mathrm{p}$ to $\mathrm{p}_{1}$, and the velocity is increased from $\mathrm{u}$ to $\mathrm{u}_{1}$ (supersonic). A local low pressure appears in the nozzle and the ejected steam is sucked. Driven by the pressure difference, the ejected steam is drawn into the suction chamber. Under the action of the viscous force, the expanding core of the working steam 
introduces the injected steam into the mixing chamber of the jet pump and accelerates it, and the two airflows perform energy exchange in the mixing. The working steam at the rear of the chamber is completely mixed with the ejected steam to reach the same speed $\mathrm{u}_{3}$, and the pressure also rises from $\mathrm{p}_{2}$ to $\mathrm{p}_{3}$. Then, the mixed fluid generates a positive shock at a section of the throat and produces a compression effect on the mixed steam. The speed of the mixed fluid is suddenly reduced from the supersonic speed to $\mathrm{u}_{3}$ (subsonic speed), and the pressure value also has a step increase from $\mathrm{p}_{3}$ to $\mathrm{p}_{4}$. Finally, the mixed gas exits from the injector. A multi-stage injection pump is made up of a series multiple injectors. The pumped gas is compressed step by step at the injector. At the last level (i.e. atmospheric level), the pressure is higher than the atmospheric pressure and is discharged from the jet pump [5].

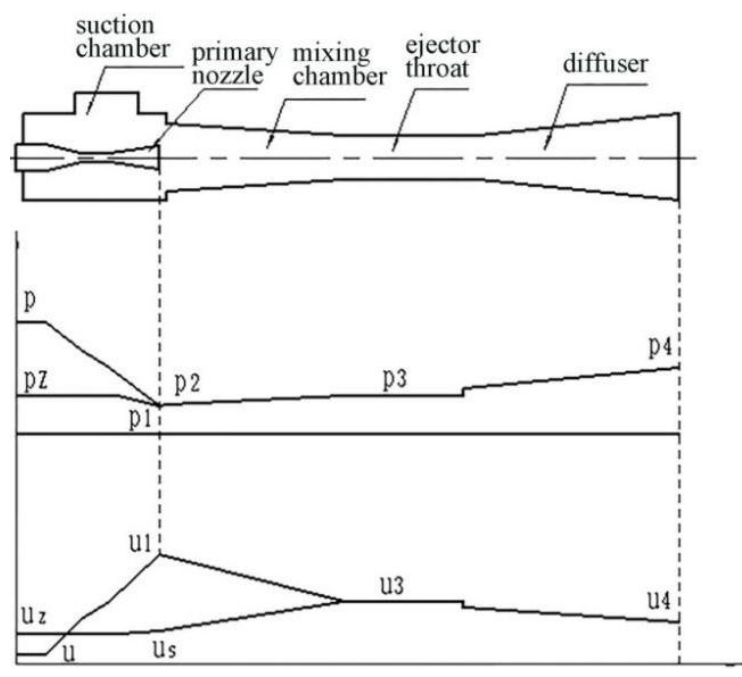

Figure 1. The working principle of the steam ejector

\subsection{Shock effect in steam ejectors}

In the steam ejector, the shock effect has a significant effect on the working performance of the ejector. Steam does the compressional expansion in the nozzle, forming a lower pressure than the ejected fluid at the outlet of the nozzle. Therefore, after the supersonic air flows out of the nozzle, it will be subjected to high back pressure compression to form an oblique shock wave. Because the oblique shock wave at the outlet of the nozzle can normally intersect at the center, and the compression wave is formed again, the air flow is compressed twice, so the Mach number is reduced, and the pressure rises to a level higher than the surrounding medium. As a result, the gas flow generates an expansion wave at the periphery of the boundary between the compression wave and the boundary layer. and then the expansion wave intersects again to form the expansion wave. After the air flow expands twice, the velocity increases and the pressure decreases, usually lower than the ambient pressure [6]. As a result, in the course of its continued flow, it must be oppressed by the surrounding medium, and the compression wave is formed again on the boundary layer. The wave is reflected by the boundary layer and multiple reflections form a very regular diamond grid pattern [5], as shown in Figure 2. Between the flow area of the working steam and the flow area of the periphery of the ejected steam, there is a layer with a large velocity gradient, namely a shock-mixing layer. The high-speed working steam transmits its kinetic energy to the ejected steam through the viscous-carrying effect of the shock-mixing layer to increase its speed, and the ejected steam passes through the shock mixing layer to trap the working steam, making the working steam decline gradually. At the end of the pump chamber, the velocity of the two- strand fluid is close or even common.

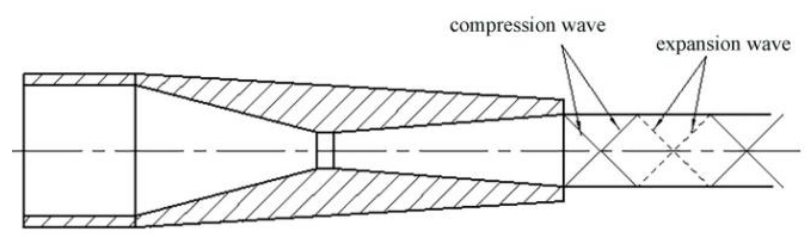

Figure 2. Supersonic ejector outlet shock effect

The flow inside the steam injection pump is very complicated, including the subsonic, sonic, and supersonic flow conditions. It is generally difficult to observe through experiments, but the flow parameters can be obtained through numerical simulation of the flow field.

\section{CFD Model and Internal Flow Simulation}

\subsection{Computational model and boundary conditions}

Injector are two-dimensional axisymmetric structures, except that the suction inlet is often non-axisymmetrically arranged so that the flow of suction fluid into the injector is three-dimensional. Considering that the gas flow velocity in the inlet region of the ejection fluid is very small compared with the flow rate of the working gas at the outlet of the nozzle, so the lateral inlet of the ejection fluid can be simplified into an axial annular inlet. Thus, the three-dimensional structure of the injector is simplified into a two-dimensional axisymmetric model. The mesh is properly encrypted at the nozzle, and grids are refined for areas with large velocity gradients and pressure gradients[7,8].

In this paper, the inlet steam and ejector steam are both considered saturated and are assumed as ideal gas. The calculation uses pressure inlet and outlet boundary conditions. Turbulence model uses Realizable k- $\varepsilon$ model. The wall surface of the injector is set to a non-slip condition, and the near wall surface treatment mode is set to a standard wall surface function. In the calculation, all residual items are below $10^{-6}$, and the mass flow is stable[8,9]. 


\subsection{Internal flow field simulation}

In the simulation, the working steam pressure is $\mathrm{p}_{\mathrm{p}}=0.2 \mathrm{Mpa}$, the ejected steam pressure is $\mathrm{p}_{\mathrm{h}}=0.02 \mathrm{Mpa}$, and the working back pressure is $p_{c}=0.035 \mathrm{Mpa}$.

The Mach cloud diagram of the flow field inside the ejector is shown in Figure 4. It can be seen from Figure 4 that the working fluid forms a supersonic high-speed jet after it is ejected through the nozzle. There is a velocity gradient between the high-speed jet and the low-velocity jet and momentum exchange occurs. The result of the momentum transformation accelerates the flow of the low-speed ejector fluid, decelerates the high-speed jet, and fully develops the fluid mixture in the mixing chamber. The two fluids become more and more uniform in speed, completing the mixing process. The velocity of the mixed fluid in the diffuser chamber is further reduced, the dynamic pressure is converted into static pressure, and the pressure is increased to the back pressure requirement.

Figures 4 and 5 are the Mach numbers and pressure distribution along the axis of the fluid inside the ejector. It can be seen from the figure that after the high-pressure working fluid passes through the nozzle, the speed rises sharply along the axis. At the outlet of the nozzle, the Maher number is over 2.5, the supersonic speed is reached, the pressure is rapidly reduced, the local low pressure area is formed, sucks the injection fluid to flow forward. The intermediate high-speed fluid collide with the two sides of the low-speed fluid, mix and transmit energy, and the two form the speed change rule of the change. Finally, the speed tends to be consistent. After the working fluid passes through the nozzle, the pressure decreases and the speed changes, resulting in an oblique shock wave. Thus, it was verified that the supersonic jet of the nozzle undergoes the compression wave, and the expansion wave repeatedly compresses and expands the shock wave characteristics. In the diffusion section of the ejector, the mixed fluid is disturbed to produce a positive shock, resulting in a sharp drop in the velocity and the pressure rising to the back pressure.

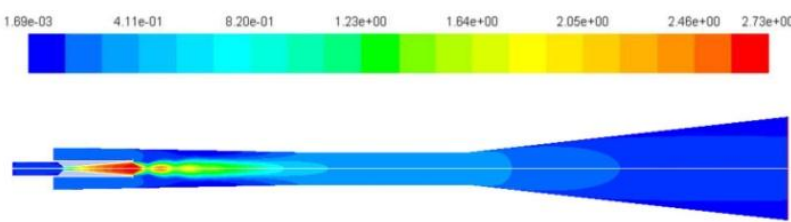

Figure 3. Mach number internal cloud of ejector

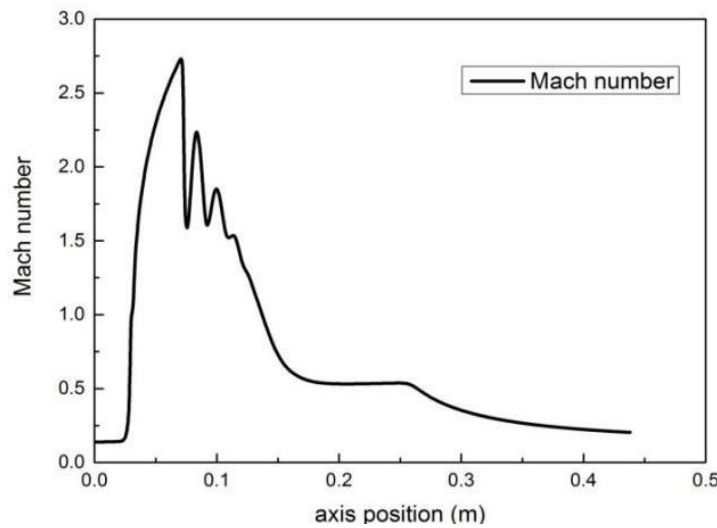

Figure 4. Mach number distribution along the axis

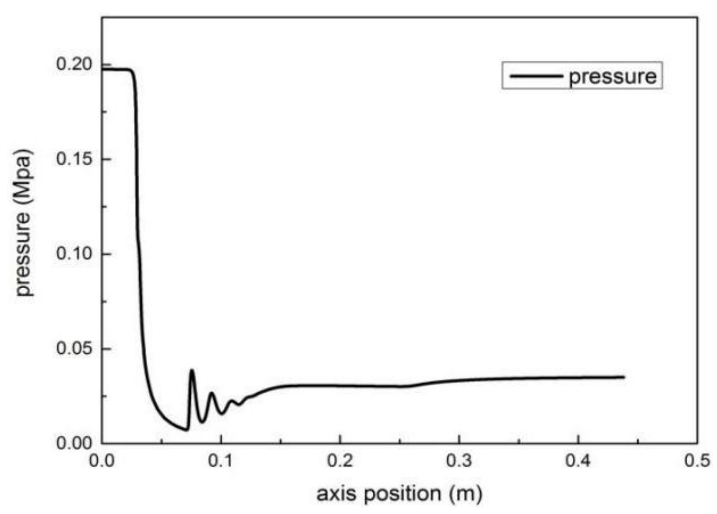

Figure 5. Pressure distribution along the axis

\section{Analysis of shock wave characteristics in steam ejectors}

\subsection{Influence of back pressure on shock wave characteristics}

In the simulation process, the working steam is ensured, and the inlet pressure of the stimulating steam does not change and the working back pressure is changed. Work back pressure $\mathrm{p}_{\mathrm{c}}$ varies between $0.02 \mathrm{Mpa}$ and $0.04 \mathrm{Mpa}$.

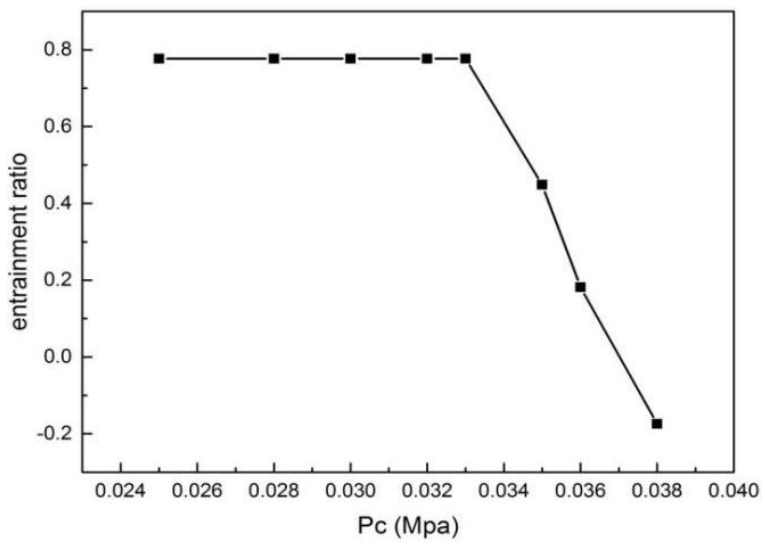

Figure 6. The entrainment ratio varies with $p_{c}$

Figure 6 shows the variation of the entrainment ratio $u$ with the back pressure $p_{c}$. Obviously seen from the figure. entrainment ratio basically remains unchanged in the 
initial stage, when the back pressure is greater than a certain value, entrainment ratio drops abruptly, so the critical back pressure $p_{c r}$ exists in the steam ejector. When $\mathrm{p}_{\mathrm{c}}<\mathrm{p}_{\mathrm{cr}}$, entrainment ratio basically remains unchanged, at this time the steam ejector is in a stable working state; when $p_{c}>p_{c r}$, entrainment ratio decreases rapidly with $p_{c}$, and even reflux occurs. At this point, the working performance of the injector gradually deteriorated.

Figure 7 presents the Mach cloud under different mixed vapor pressures $p_{c}$. It can be clearly seen from the cloud image that a double shock wave phenomenon has appeared in the steam ejector. The first shock wave occurs near the nozzle exit section. The second shock wave appears in the throat section of the steam ejector or in the inlet section of the diffuser chamber. This position is closely related to the total energy of the mixed fluid. When the fluid energy is high, the second shock wave moves toward the entrance of the diffusion chamber, and the injector can work at a higher critical back pressure.

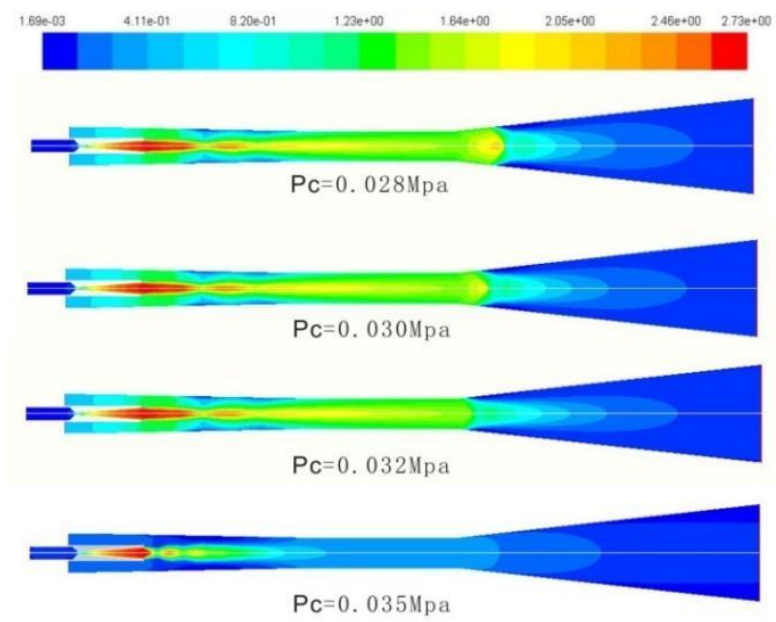

Figure 7. Maher number cloud under different $p_{c}$

Figure 8 presents the distribution of Mach numbers along the axis under different $p_{c}$. It can be clearly seen from the figure that when $\mathrm{p}_{\mathrm{c}}<\mathrm{p}_{\mathrm{cr}}$, the change of $\mathrm{p}_{\mathrm{c}}$ has less influence on the first shock wave and can be ignored, but it has a greater impact on the second shock wave. When the back pressure is vary from $0.028 \mathrm{Mpa}$ to $0.032 \mathrm{Mpa}$, the shock wave characteristics appear at the inlet of the diffuser chamber, which is reflected in the compression shock characteristic of pressure step increase and speed step decrease. Steam velocity decreases from supersonic to subsonic. The working fluid and the ejecting fluid are choked at the injector's main nozzle and the diffuser inlet so that the flow of working fluid and ejector fluid does not change with the outlet pressure. Therefore, entrainment ratio remains unchanged, and the position of the second shock wave increases as $p_{c}$ moves toward the upstream of the diffuser. When $\mathrm{p}_{\mathrm{c}}>\mathrm{p}_{\mathrm{cr}}$, the mixing action of the fluid causes the position of the second shock wave to move toward the mixing chamber. The shock wave at this time interferes with the weakening or even elimination of the jamming phenomenon of the effective mixing of the two fluids at the inlet of the diffuser chamber. As a result, a small amount of ejected fluid is sucked into the ejector and the entrainment ratio decreases. When the $\mathrm{p}_{\mathrm{c}}$ continues to rise, and reaches or exceeds the back pressure, the second shock wave moves toward the nozzle. At this point, the working fluid is disturbed by it, this no longer produces swell wave even produces reflux phenomenon.

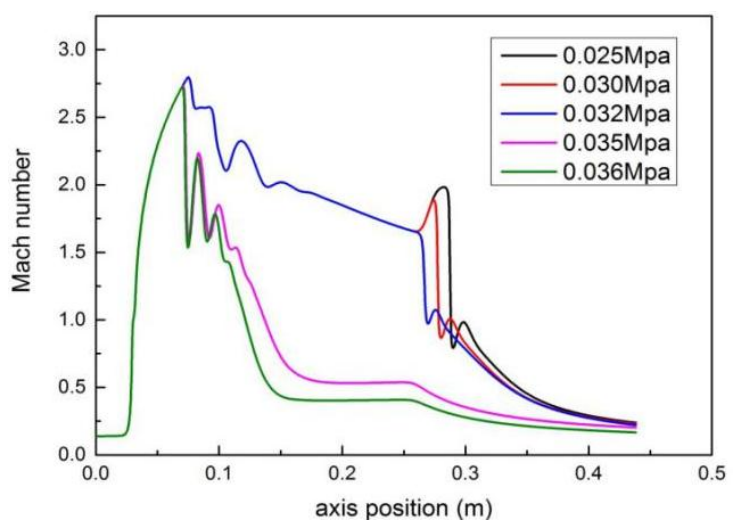

Figure 8. Distribution of Mach numbers along the axis under different $p_{c}$

\subsection{Effect of throat diameter on shock wave characteristics}

The working fluid reaches the supersonic velocity at the outlet of the nozzle, and the Mach number at the nozzle outlet cross section is determined by the ratio of the nozzle outlet sectional area to the throat sectional area. The Mach number at the outlet of the nozzle is also a very important parameter reflecting the working capability of the injector model. Therefore, changing the diameter of the nozzle throat, examining the influence of the diameter of the throat on the shock characteristics and the influence on the ejection coefficient is particularly important.

In the simulation, the working steam pressure is $p_{p}$ $=0.2 \mathrm{Mpa}$, the ejected steam pressure is $\mathrm{p}_{\mathrm{h}}=0.02 \mathrm{Mpa}$, and the working back pressure is $p_{c}=0.035 \mathrm{Mpa}$. Keep the diameter of the nozzle outlet constant, adjust the diameter of the nozzle throat $\mathrm{d}$, and observe the effect of changes in the structure of the injector on the nozzle outlet flow field. Figure 9 shows the relationship between the entrainment ratio and the diameter of the throat of the nozzle. The diameter of the throat of the nozzle has a great influence on the entrainment ratio. For a specific design condition, there is an optimal nozzle throat diameter. 


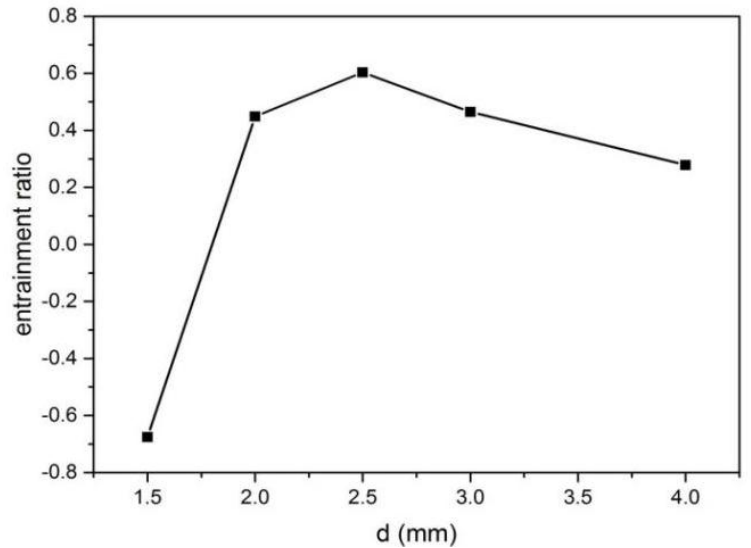

Figure 9. Distribution of Mach numbers along the axis under different $p_{c}$

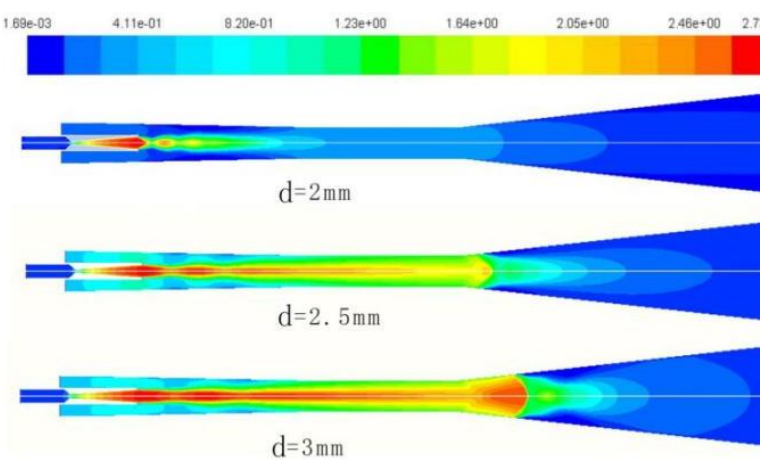

Figure 10. Mach Clouds with different diameters

Figure 10 shows the Mach number in the throat diameter of different nozzles. It can be clearly seen from the figure, the difference in the internal flow field of the different throat diameter injectors. The shock waves generated mainly by the supersonic fluid at the outlet of the nozzle are different and the distribution of the flow field in the diffusion chamber is different. When the nozzle throat diameter is small, two kinds of fluid in the diffuser entrance choking phenomenon is very weak or there is no second shock wave, resulting in a lower entrainment ratio and even backflow. When the nozzle throat diameter is large, the two fluids are not completely mixed in the mixing chamber, the speed and pressure have strong fluctuations, resulting in greater loss of mechanical energy. By observing the shape and length of the shock wave, it can be seen that when the shock wave is generated at the outlet of the nozzle and extends to the vicinity of the outlet of the mixing section, the entrainment ratio reaches the highest, which is the best state of the ejector performance. When the shock wave ends prematurely or belatedly, it affects the performance of the injector.

\section{Conclusion}

In this paper, numerically simulates the complex flow of the flow field inside the injector. By changing the working back pressure of the steam ejector and the diameter of the throat of the nozzle, the internal shock characteristics of the steam ejector are analyzed and the influence of shock variation on the overall content of the ejector is studied. Get the following conclusions:

Verify that the steam ejector working fluid generates supersonic fluid through the laval nozzle, and generate a series of shock trains inside the injector. The location of the shock wave generation and termination has a great influence on the ejector performance.

There is a critical back pressure inside the steam ejector. When the working back pressure is lower than the critical back pressure, the entrainment ratio remains unchanged, and the working fluid and the ejecting fluid generate shock waves at the main nozzle of the injector and the entrance of the diffuser chamber, causing a jamming phenomenon. With the increase of the back pressure, the position of the second shock moves toward the upstream of the diffuser. When the working back pressure is higher than the critical back pressure, the position of the second shock wave moves to the mixing chamber as the back pressure increases. The jamming phenomenon at the inlet of the diffuser is weakened or even relieved, the entrainment ratio is drastically reduced, and the ejector even returns.

The Mach number at the nozzle outlet cross section of the steam ejector has a large relationship with the nozzle throat diameter, and the nozzle throat diameter has an optimal value. When the diameter of the throat of the nozzle is small, the congestion at the inlet of the diffuser chamber is very weak and there is no second shock wave, and the entrainment ratio is low. When the throat diameter of the nozzle is larger, the energy loss is higher and the entrainment ratio is low. When the nozzle diameter is optimal, the shock wave is generated by the nozzle outlet and ends when it reaches near the outlet of the mixing section. The ejection entrainment ratio the maximum.

\section{References}

1. X. D. Wang, J. L. Dong, J. Y. Tu. Numerical analysis of the effect of suction pressure on pumping performance of steam injectionpump [J]. Journal of Vacuum Science and Technology, 30, 695-698 (2010).

2. X. Z. Wu, S. Xia, J. Qiu. CFD Analysis of Internal flow Field and Variable Conditions of Supersonic Jet[J]. Machinery Design \& Manufacture, 09, 49-51 (2014).

3. Shah A, Chughtai I R, Inayat M H. Experimental and numerical investigation of the effect of mixing section length on direct-contact condensation in steam jet pump[J]. International Journal of Heat \& Mass Transfer, 72, 430-439 (2014).

4. H. Wu, Z. Liu, B. Han, et al. Numerical investigation of the influences of mixing chamber geometries on steam ejector performance[J]. Desalination, 353, 15-20 (2014).

5. Oishi T, Watanabe T, Undagawa Y. Mixing Characteristics for a Supersonic Ejector[J]. ASME-FED, High Speed Jet Flow, 214, 157-162 (1995). 
6. Y. Yang, Y. Q. Li, S. Q. Shen, P. Li, et al. Shock wave effects in steam ejectors[J]. Journal of Engineering Thermophysics, 35, 1419-1423 (2014).

7. J. Yao. CFD numerical simulation of steam injectors and process state analysis [D]. North China Electric Power University (Beijing), 2009.

8. H. J. Li, S. Q. Shen, B. Zhang. Numerical simulation of two-dimensional flow field in steam ejector[J]. Thermal Science and Technology, 04, 353-357 (2004).

9. D. A. Da. Vacuum Design Manual [M]. National Defense Industry Press, 2004. 